PROCEEDINGS OF THE

AMERICAN MATHEMATICAL SOCIETY

Volume 139, Number 2, February 2011, Pages 521-534

S 0002-9939(2010)10490-2

Article electronically published on July 16, 2010

\title{
LYUSTERNIK-GRAVES THEOREM AND FIXED POINTS
}

\author{
ASEN L. DONTCHEV AND HÉLÈNE FRANKOWSKA
}

(Communicated by Nigel J. Kalton)

\begin{abstract}
For set-valued mappings $F$ and $\Psi$ acting in metric spaces, we present local and global versions of the following general paradigm which has roots in the Lyusternik-Graves theorem and the contraction principle: if $F$ is metrically regular with constant $\kappa$ and $\Psi$ is Aubin (Lipschitz) continuous with constant $\mu$ such that $\kappa \mu<1$, then the distance from $x$ to the set of fixed points of $F^{-1} \Psi$ is bounded by $\kappa /(1-\kappa \mu)$ times the infimum distance between $\Psi(x)$ and $F(x)$. From this result we derive known Lyusternik-Graves theorems, a recent theorem by Arutyunov, as well as some fixed point theorems.
\end{abstract}

\section{LyUSTERNiK-GRAVES THEOREM}

The concept of metric regularity and its sibling's openness with linear rate and Aubin continuity have their roots in the Banach open mapping principle which, according to the original treatise of Banach [3], p. 150, says that the surjectivity of a linear and bounded mapping $A$, acting from a Banach space $(X,\|\cdot\|)$ to a Banach space $(Y,\|\cdot\|)$, is equivalent to the existence of a positive constant $\kappa$ such that for any $y \in Y$ there exists $x \in X$ with $A x=y$ and also $\|x\| \leq \kappa\|y\|$. In terms of the distance $d(x, C)$ from a point $x$ to a set $C$ the latter condition is equivalent to $d\left(x, A^{-1}(y)\right) \leq \kappa\|y-A x\|$ for all $(x, y) \in X \times Y$, a property of the mapping $A$ which is nowadays known as metric regularity.

The Banach open mapping theorem has been extended to nonlinear mappings in the work of Graves [13] who obtained the following result: let $f: X \rightarrow Y$ be a function which is continuous in a neighborhood of $\bar{x}$, let $A: X \rightarrow Y$ be a surjective linear and bounded mapping, and let $\kappa$ be the constant in the Banach principle for the mapping $A$. Let $f-A$ be Lipschitz continuous in a ball $\mathbb{B}_{\varepsilon}(\bar{x})$ centered at $\bar{x}$ with radius $\varepsilon>0$ with Lipschitz constant $\mu$ such that $\kappa \mu<1$. Then, in terms of $\bar{y}=f(\bar{x})$, if $y$ satisfies $\|y-\bar{y}\| \leq\left(\kappa^{-1}-\mu\right) \varepsilon$, then the equation $f(x)=y$ has a solution $x$ in $\mathbb{B}_{\varepsilon}(\bar{x})$. A slight modification in the original proof of Graves (see 9 , p. 277]) gives us the condition

$$
d\left(x, f^{-1}(y)\right) \leq \frac{\kappa}{1-\kappa \mu}\|y-f(x)\| \quad \text { for any }(x, y) \text { near }(\bar{x}, \bar{y}),
$$

which is the definition of local metric regularity of a function $f$ at $\bar{x}$ for $\bar{y}$.

Received by the editors December 24, 2009 and, in revised form, March 08, 2010.

2010 Mathematics Subject Classification. Primary 49J53; Secondary 47J22, 49J40, 49K40, 90C31.

Key words and phrases. Metric regularity, openness, Lyusternik-Graves theorem, fixed point, Ekeland principle.

The first author was supported by National Science Foundation grant DMS 1008341.

(C) 2010 American Mathematical Society Reverts to public domain 28 years from publication 
The next important step in generalizing the Banach principle is due to Milyutin (see [5]), who observed that the mapping $A$ in Graves' theorem can be replaced by any function $h$ which is open with linear rate, a property that turned out to be equivalent to metric regularity. In terms of metric regularity, Milyutin's theorem says that if a function $h$ is metrically regular at $\bar{x}$ for $\bar{y}=h(\bar{x})$ with constant $\kappa$ and, for a function $f$ with $f(\bar{x})=h(\bar{x})=\bar{y}$, the difference $f-h$ is Lipschitz continuous with constant $\mu$ such that $\kappa \mu<1$, then $f$ is metrically regular at $\bar{x}$ for $\bar{y}$ with constant $\kappa /(1-\kappa \mu)$, as in (1). Milyutin and his coauthors [5] linked this result with a theorem by Lyusternik [16], which characterizes the tangent manifold to the kernel of a function at a given point, a result weaker than Graves' theorem. One should note that both Lyusternik and Graves, as well as Milyutin, used in their proofs iterative schemes that resemble the Picard iteration or, even more directly, the Newton method.

In the last several decades, metric regularity has been recognized as a basic property in the general area of optimization, which serves as a major constraint qualification condition 1 in deriving optimality conditions, and even more importantly, is very instrumental in obtaining error bounds for perturbed minima and proving convergence of algorithms for solving optimization problems and beyond. For a comprehensive treatment of these developments together with historical remarks, see the recent book [9], as well as the earlier survey [14.

To proceed, let us first fix the notation. Throughout $f: X \rightarrow Y$ means that $f$ is a function (a single-valued mapping) while $F: X \rightrightarrows Y$ denotes a general mapping which may be set-valued. The graph of $F$ is the set $\operatorname{gph} F=\{(x, y) \in X \times Y \mid y \in$ $F(x)\}$, and the inverse of $F$ is the mapping $y \mapsto F^{-1}(y)=\{x \mid y \in F(x)\}$. The closed ball centered at $x$ with radius $r$ is $\mathbb{B}_{r}(x)$ while the open one is $\stackrel{\circ}{B}_{r}(x)$; when $X$ is a linear metric space, the closed unit ball centered at zero is $\mathbb{B}$ and the open one is $\stackrel{\circ}{\mathbb{B}}$. By convention, for $x \in X, \mathbb{B}_{\infty}(x)=X$. A set $C$ is said to be locally closed (resp., complete) at $\bar{x} \in C$ when there exists $r>0$ such that $C \cap \mathbb{B}_{r}(\bar{x})$ is closed (resp., complete). The metric in a metric space $X$ is denoted by $d_{X}$. The distance from a point $x$ to a nonempty set $C$ in a metric space $\left(X, d_{X}\right)$ is denoted by $d(x, C) ; d(x, C)=\inf _{v \in C} d_{X}(x, v)$. We set $d(x, C)=\infty$ iff $C=\emptyset$. The excess from a set $C$ to a set $D$ is $e(C, D)=\sup _{x \in C} d(x, D)$ and the PompeiuHausdorff distance between the sets $C$ and $D$ is $h(C, D)=\max \{e(C, D), e(D, C)\}$. The infimum distance between points of two sets $C$ and $D$ in $\left(X, d_{X}\right)$ is denoted by $d(C, D) ; d(C, D)=\inf _{x \in C, v \in D} d_{X}(y, v)$. Given a mapping $\Phi: X \rightrightarrows X$, we denote $\operatorname{Fix}(\Phi)=\{x \in X \mid x \in \Phi(x)\}$.

The definition of metric regularity of a general set-valued mapping is as follows:

Definition 1 (metric regularity). Given two metric spaces $\left(X, d_{X}\right)$ and $\left(Y, d_{Y}\right)$, a mapping $F: X \rightrightarrows Y$ is said to be metrically regular at $\bar{x}$ for $\bar{y}$ when $\bar{y} \in F(\bar{x})$ and there is a constant $\kappa>0$ together with neighborhoods $U$ of $\bar{x}$ and $V$ of $\bar{y}$ such that

$$
d\left(x, F^{-1}(y)\right) \leq \kappa d(y, F(x)) \text { for all }(x, y) \in U \times V .
$$

The infimum of $\kappa$ over all such combinations of $\kappa, U$ and $V$ is called the regularity modulus for $F$ at $\bar{x}$ for $\bar{y}$ and is denoted by $\operatorname{reg}(F ; \bar{x} \mid \bar{y})$.

${ }^{1}$ The main aim of Lyusternik in $[16$ was deriving a Lagrange multiplier rule for an abstract optimization problem with equality constraints. 
It is now well known that the metric regularity is equivalent to two other properties. The first one is the openness with linear rate defined as follows: A mapping $F: X \rightrightarrows Y$ is said to be open with linear rate or linearly open at $\bar{x}$ for $\bar{y}$ when $\bar{y} \in F(\bar{x})$ and there are constants $\rho>0$ and $h_{0}>0$ together with neighborhoods $U$ of $\bar{x}$ and $V$ of $\bar{y}$ such that

$$
\stackrel{\circ}{B}_{h \rho}(y) \subset F\left(\stackrel{\circ}{B}_{h}(x)\right) \text { for all }(x, y) \in \operatorname{gph} F \cap(U \times V), h \in\left(0, h_{0}\right) .
$$

It is important to note that while the neighborhoods $U$ and $V$ in (2) and (3) may be different, the constant $\rho$ in (3) could be any positive number smaller than $1 / \operatorname{reg}(F ; \bar{x} \mid \bar{y})$. The standard openness, that is, the property that $F(U)$ is open when $U$ is open, is implied by (3), but the converse is not true. Linear openness postulates openness around the reference point with balls having proportional radii. In the literature the openness with linear rate is sometimes called the covering property; e.g., a mapping $F$ is open with rate $\rho$ if it is covering with constant $\rho$.

The second equivalent property is the Aubin continuity 2 A mapping $\Psi: X \rightrightarrows Y$ is said to be Aubin continuous at $\bar{x}$ for $\bar{y}$ when there are a constant $\kappa$ and neighborhoods $U$ of $\bar{x}$ and $V$ of $\bar{y}$ such that

$$
e\left(\Psi(x) \cap V, \Psi\left(x^{\prime}\right)\right) \leq \kappa d_{X}\left(x, x^{\prime}\right) \text { for all } x, x^{\prime} \in U .
$$

The infimum of $\kappa$ over all $\kappa, U$ and $V$ for which (4) holds is denoted $\operatorname{Lip}(\Psi ; \bar{x} \mid \bar{y})$. It turns out that a mapping $F$ is metrically regular at $\bar{x}$ for $\bar{y}$ with constant $\kappa$ (or, equivalently, linearly open with rate $1 / \kappa)$ if and only if its inverse $F^{-1}$ is Aubin continuous at $\bar{y}$ for $\bar{x}$; moreover, $\operatorname{Lip}\left(F^{-1} ; \bar{y} \mid \bar{x}\right)=\operatorname{reg}(F ; \bar{x} \mid \bar{y})$. If $\Psi$ in (4) is single-valued, say, a function $s$ with $s(\bar{x})=\bar{y}$, then (4) simply means that $s$ is Lipschitz continuous near $\bar{x}$, and then $\operatorname{Lip}(s ; \bar{x} \mid s(\bar{x}))$ equals the Lipschitz modulus of $s$, denoted $\operatorname{lip}(s ; \bar{y})$, which is the lower limit of the Lipschitz constants of $s$ taken over all neighborhoods of $\bar{x}$.

If $V=Y$ in (4), then the mapping $\Psi$ becomes Lipschitz continuous in $U$ with respect to the Pompeiu-Hausdorff distance; that is,

$$
h\left(\Psi(x), \Psi\left(x^{\prime}\right)\right) \leq \kappa d_{X}\left(x, x^{\prime}\right) \text { for all } x, x^{\prime} \in U .
$$

If in addition $U=X$, we say that the mapping $\Psi$ is globally Lipschitz continuous.

We will also need a partial version of the Aubin continuity. A mapping $\Psi$ : $P \times X \rightrightarrows Y$ is said to be partially Aubin continuous with respect to $x$ uniformly in $p$ at $(\bar{p}, \bar{x})$ for $\bar{y}$ if $\bar{y} \in \Psi(\bar{p}, \bar{x})$ and there is a nonnegative constant $\kappa$ together with neighborhoods $Q$ for $\bar{p}, U$ of $\bar{x}$ and $V$ of $\bar{y}$ such that

$$
e\left(\Psi(p, x) \cap V, \Psi\left(p, x^{\prime}\right)\right) \leq \kappa d_{X}\left(x, x^{\prime}\right) \quad \text { for all } x, x^{\prime} \in U \text { and } p \in Q .
$$

The infimum of $\kappa$ over all such combinations of $\kappa, Q, U$ and $V$ is called the partial Lipschitz modulus of $\Psi$ with respect to $x$ uniformly in $p$ and is denoted by $\widehat{\operatorname{Lip}}_{x}(\Psi ;(\bar{p}, \bar{x}) \mid \bar{y})$.

For $U=X$ and $V=Y$, Definition 1 gives us global metric regularity, in which the reference point $(\bar{x}, \bar{y})$ can be dropped. Also, the property in (3) becomes global linear openness or a global covering, while, as already mentioned, Aubin continuity (4) reduces to global Lipschitz continuity. As in the local case, these three global properties are equivalent to each other (for the Lipschitz continuity of the inverse mapping). The global properties are quite different from their local counterparts, and we will see the difference more explicitly in what follows.

\footnotetext{
${ }^{2}$ Originally introduced in 2 under the name pseudo-Lipschitz continuity.
} 
We recall next a result generalizing the theorems of Lyusternik, Graves and Milyutin, in a form which slightly extends Theorem 5E.1 in [9]. Following [14], we call this theorem the extended Lyusternik-Graves theorem:

Theorem 1 (Extended Lyusternik-Graves). Let $\left(X, d_{X}\right)$ be a metric space, $\left(Y, d_{Y}\right)$ be a linear metric space with shift-invariant metric $d_{Y}$. Consider a mapping $F$ : $X \rightrightarrows Y$, a point $(\bar{x}, \bar{y}) \in \operatorname{gph} F$ and a function $g: X \rightarrow Y$. Assume that gph $F$ is locally complete at $(\bar{x}, \bar{y})$. Also, assume that $F$ is metrically regular at $\bar{x}$ for $\bar{y}$, that $g$ is Lipschitz continuous in a neighborhood of $\bar{x}$, and that there exist constants $\kappa$ and $\mu$ such that

$$
\kappa \mu<1, \quad \operatorname{reg}(F ; \bar{x} \mid \bar{y}) \leq \kappa \quad \text { and } \quad \operatorname{lip}(g ; \bar{x}) \leq \mu .
$$

Then the mapping $g+F$ is metrically regular at $\bar{x}$ for $g(\bar{x})+\bar{y}$ with

$$
\operatorname{reg}(g+F ; \bar{x} \mid g(\bar{x})+\bar{y}) \leq \frac{\kappa}{1-\kappa \mu} .
$$

In the following section we will derive Theorem 1 from our main results. In Section 3, for the case when $Y$ is a normed space, we will supply Theorem 1 with a new proof based on the Ekeland variational principle. A different kind of extension of the Lyusternik-Graves/Milyutin theorem was recently given in [15].

Theorem 1 was supplied in [9] with two separate proofs: the first proof uses a Picard/Newton type iteration resembling the original argument in the proofs of Lyusternik, Graves and Milyutin, while the second proof is based on the following fixed point theorem for set-valued mappings proved in 8]; see also [9, Theorem 5E.2]:

Theorem 2. Let $\left(X, d_{X}\right)$ be a complete metric space and consider a set-valued mapping $\Phi: X \rightrightarrows X$ and a point $\bar{x} \in X$. Suppose that there exist scalars $c>0$ and $\lambda \in(0,1)$ such that the set gph $\Phi \cap\left(\mathbb{B}_{c}(\bar{x}) \times \mathbb{B}_{c}(\bar{x})\right)$ is closed and

(a) $d(\bar{x}, \Phi(\bar{x}))<c(1-\lambda)$;

(b) $e\left(\Phi(u) \cap \mathbb{B}_{c}(\bar{x}), \Phi(v)\right) \leq \lambda d_{X}(u, v)$ for all $u, v \in \mathbb{B}_{c}(\bar{x})$.

Then $\Phi$ has a fixed point in $\mathbb{B}_{c}(\bar{x})$; that is, there exists $x \in \mathbb{B}_{c}(\bar{x})$ such that $x \in \Phi(x)$.

Note that for $c=\infty$, Theorem 2 reduces to the well-known Nadler fixed point theorem [17.

Observe that the statement of Theorem 1 can be rewritten as

$$
\begin{array}{r}
\inf _{g: X \rightarrow Y}\{\operatorname{lip}(g ; \bar{x}) \mid F+g \text { is not metrically regular } \\
\text { at } \bar{x} \text { for } \bar{y}+g(\bar{x})\} \geq \frac{1}{\operatorname{reg}(F ; \bar{x} \mid \bar{y})} .
\end{array}
$$

In other words, Theorem 1 gives a lower bound for the quantity which measures the "distance" from a metrically regular mapping to the set of mappings that are not regular; that is, it gives a lower estimate for the radius of metric regularity. It was proven in [10] that if, in addition to the assumptions of Theorem 1, $X$ and $Y$ are finite-dimensional spaces, then the inequality $\geq$ in (5) becomes equality. Moreover, in this case the infimum in (5) is unchanged if the class of perturbations is reduced to linear bounded mappings of rank 1 . It is still an open question to characterize classes of infinite-dimensional spaces and mappings acting in them for which (5) holds as equality. 
Another important observation is that Theorem 1 cannot be extended to cover the case when the mapping $g$ is set-valued, as shown by a counterexample in 9, p. 291. If, however, Theorem 1 is restated for global metric regularity, then it would also cover the case when both $F$ and $g$ are set-valued. Such a result can be easily derived from [12, Theorem 1.1] and is stated in various ways in [14, 6] and [7. Here we adopt it in the following form:

Theorem 3 (Global Lyusternik-Graves). Let $\left(X, d_{X}\right)$ be a metric space and let $\left(Y, d_{Y}\right)$ be a linear metric space with shift-invariant metric $d_{Y}$. Consider mappings $F: X \rightrightarrows Y$ and $G: X \rightrightarrows Y$ and assume that one of the sets $\operatorname{gph} F$, gph $G$ is complete while the other is closed. Let $\kappa$ and $\mu$ be nonnegative constants such that $\kappa \mu<1$ and suppose that $F$ is metrically regular with constant $\kappa$ and that $G$ is Lipschitz continuous with constant $\mu$, both globally. Then $F+G$ is globally metrically regular with constant $\kappa /(1-\kappa \mu)$.

Arutyunov published recently in [1] the following coincidence theorem and showed that it implies both Theorem 3 and the Nadler fixed point theorem:

Theorem 4 (Arutyunov [1). Let $\left(X, d_{X}\right)$ and $\left(Y, d_{Y}\right)$ be metric spaces. Consider a mapping $F: X \rightrightarrows Y$ with closed graph which satisfies for some $\gamma>0$,

$$
\mathbb{B}_{\gamma h}(F(x)) \subset F\left(\mathbb{B}_{h}(x)\right) \text { for all } h \geq 0 \text { and } x \in X .
$$

Consider also a closed-valued mapping $\Psi: X \rightrightarrows Y$ which is globally Lipschitz continuous with Lipschitz constant $\mu<\gamma$. Let either gph $F$ or gph $\Psi$ be complete. Then for any $x \in X$ and $\varepsilon>0$ there exists $\xi$ such that

$$
F(\xi) \cap \Psi(\xi) \neq \emptyset \quad \text { and } \quad d_{X}(\xi, x) \leq \frac{1}{\gamma-\mu} d(F(x), \Psi(x))+\varepsilon .
$$

Condition (6) for the mapping $F$ is clearly equivalent to the global linear openness, i.e., the property in (3) with $U=X, V=Y$ and $h_{0}=\infty$, which is equivalent to the global metric regularity with constant $1 / \gamma$. The main result in this paper, which is given in the following section, is a generalization of Theorem 4 that covers both the local and the global cases, as well as all other theorems given above.

\section{Main Results And CONSEquences}

In this section we will present our main result, Theorem 5, as well as two more elaborate versions of it, from which we will derive all theorems given in Section 1.

Theorem 5. Let $\left(X, d_{X}\right),\left(Y, d_{Y}\right)$ be metric spaces and consider two mappings $F: X \rightrightarrows Y$ and $\Psi: X \rightrightarrows Y$ and a point $(\bar{x}, \bar{y}) \in \operatorname{gph} F \cap \operatorname{gph} \Psi$. Assume that either one of the sets gph $F$ and gph $\Psi$ is locally complete at $(\bar{x}, \bar{y})$ while the other is locally closed at $(\bar{x}, \bar{y})$ or the set $\operatorname{gph}\left(F^{-1} \Psi\right)$ is locally complete at $(\bar{x}, \bar{x})$. Also, assume that $F$ is metrically regular at $\bar{x}$ for $\bar{y}$, that $\Psi$ is Aubin continuous at $\bar{x}$ for $\bar{y}$, and that there exist constants $\kappa$ and $\mu$ such that

$$
\kappa \mu<1, \quad \operatorname{reg}(F ; \bar{x} \mid \bar{y})<\kappa \quad \text { and } \quad \operatorname{Lip}(\Psi ; \bar{x} \mid \bar{y})<\mu .
$$

Then there exist neighborhoods $U$ of $\bar{x}$ and $V$ of $\bar{y}$ such that for any $x \in U$,

$$
d\left(x, \operatorname{Fix}\left(F^{-1} \Psi\right)\right) \leq \frac{\kappa}{1-\kappa \mu} d(\Psi(x) \cap V, F(x)) .
$$

If the assumptions for $F$ and $\Psi$ hold globally, then one can take $U=X$ and $V=Y$ in (8). 
Proof. The assumptions for the mappings $F$ and $\Psi$ yield the existence of a positive constant $\alpha$ such that one of the following conditions holds: (i) gph $F \cap\left(\mathbb{B}_{\alpha}(\bar{x}) \times\right.$ $\left.\mathbb{B}_{\alpha}(\bar{y})\right)$ is complete and gph $\Psi \cap\left(\mathbb{B}_{\alpha}(\bar{x}) \times \mathbb{B}_{\alpha}(\bar{y})\right)$ is closed; (ii) gph $\Psi \cap\left(\mathbb{B}_{\alpha}(\bar{x}) \times\right.$ $\left.\mathbb{B}_{\alpha}(\bar{y})\right)$ is complete and $\operatorname{gph} F \cap\left(\mathbb{B}_{\alpha}(\bar{x}) \times \mathbb{B}_{\alpha}(\bar{y})\right)$ is closed; (iii) $\operatorname{gph}\left(F^{-1} \Psi\right) \cap$ $\left(\mathbb{B}_{\alpha}(\bar{x}) \times \mathbb{B}_{\alpha}(\bar{x})\right)$ is complete. Also,

$$
d\left(x, F^{-1}(y)\right) \leq \kappa d(y, F(x)) \text { for all }(x, y) \in \mathbb{B}_{\alpha}(\bar{x}) \times \mathbb{B}_{\alpha}(\bar{y})
$$

and

$$
e\left(\Psi(x) \cap \mathbb{B}_{\alpha}(\bar{y}), \Psi\left(x^{\prime}\right)\right) \leq \mu d_{X}\left(x, x^{\prime}\right) \text { for all }\left(x, x^{\prime}\right) \in \mathbb{B}_{\alpha}(\bar{x}) .
$$

Pick any positive reals $a, b$ and $\varepsilon$ so that the following system of inequalities holds:

$$
\left\{\begin{array}{l}
\kappa(\mu+\varepsilon)+\varepsilon<1, \\
\frac{1}{1-(\kappa(\mu+\varepsilon)+\varepsilon)}[a+\kappa b+\varepsilon]+a \leq \alpha \\
\frac{1}{1-(\kappa(\mu+\varepsilon)+\varepsilon)}[(\mu+\varepsilon)(a+\kappa b+\varepsilon)]+b \leq \alpha .
\end{array}\right.
$$

We will now prove that (8) holds with $U=\mathbb{B}_{a}(\bar{x})$ and $V=\mathbb{B}_{b}(\bar{y})$. Fix $x \in$ $\mathbb{B}_{a}(\bar{x})$. If $\Psi(x) \cap \mathbb{B}_{b}(\bar{y})=\emptyset$, then the right side of $(8)$ (with $V=\mathbb{B}_{b}(\bar{y})$ ) is $+\infty$ and there is nothing more to prove. Let $y \in \Psi(x) \cap \mathbb{B}_{b}(\bar{y})$. From (9), there exists $z^{1} \in F^{-1}(y)$ such that

$$
d_{X}\left(z^{1}, x\right) \leq d\left(x, F^{-1}(y)\right)+\varepsilon \leq \kappa d(y, F(x))+\varepsilon .
$$

Furthermore,

$$
\begin{array}{r}
d_{X}\left(z^{1}, x\right) \leq d\left(x, F^{-1}(y)\right)+\varepsilon \leq d_{X}(x, \bar{x})+d\left(\bar{x}, F^{-1}(y)\right)+\varepsilon \\
\quad \leq a+\kappa d(y, F(\bar{x}))+\varepsilon \leq a+\kappa d_{Y}(y, \bar{y})+\varepsilon \leq a+\kappa b+\varepsilon
\end{array}
$$

Then, using (11), we also have

$$
d_{X}\left(z^{1}, \bar{x}\right) \leq d_{X}\left(z^{1}, x\right)+d_{X}(x, \bar{x}) \leq 2 a+\kappa b+\varepsilon \leq \alpha .
$$

If $z^{1}=x$, then $x \in F^{-1}(\Psi(x))$, which is the same as $x \in \operatorname{Fix}\left(F^{-1} \Psi\right)$. Then (8) holds automatically, since its left side is 0 . Let $z^{1} \neq x$. Since $d_{X}\left(z^{1}, x\right)>0$, there exists $y^{1} \in \Psi\left(z^{1}\right)$ such that

$$
d_{Y}\left(y^{1}, y\right) \leq d\left(y, \Psi\left(z^{1}\right)\right)+\varepsilon d_{X}\left(z^{1}, x\right) .
$$

From (10), remembering that $y \in \Psi(x) \cap \mathbb{B}_{b}(\bar{y})$, we obtain

$$
d_{Y}\left(y^{1}, y\right) \leq e\left(\Psi(x) \cap \mathbb{B}_{b}(\bar{y}), \Psi\left(z^{1}\right)\right)+\varepsilon d_{X}\left(z^{1}, x\right) \leq(\mu+\varepsilon) d_{X}\left(z^{1}, x\right) .
$$

Then, using (11) and (13),

$$
d_{Y}\left(y^{1}, \bar{y}\right) \leq d_{Y}(y, \bar{y})+(\mu+\varepsilon) d_{X}\left(z^{1}, x\right) \leq b+(\mu+\varepsilon)(a+\kappa b+\varepsilon) \leq \alpha .
$$

By induction, we construct sequences of points $z^{k}$ and $y^{k}$, with $z^{0}=x$ and $y^{0}=y$, such that, for $k=0,1,2, \ldots$,

$$
\begin{aligned}
& z^{k+1} \in F^{-1}\left(y^{k}\right) \text { with } d_{X}\left(z^{k+1}, z^{k}\right) \leq(\kappa(\mu+\varepsilon)+\varepsilon)^{k} d_{X}\left(z^{1}, x\right), \\
& y^{k+1} \in \Psi\left(z^{k+1}\right) \text { with } d_{Y}\left(y^{k+1}, y^{k}\right) \leq(\mu+\varepsilon) d_{X}\left(z^{k+1}, z^{k}\right) .
\end{aligned}
$$

Taking into account (15), we see that $z^{1}$ and $y^{1}$ satisfy (17) for $k=0$. Suppose that for some $n \geq 1$ we have generated $z^{1}, z^{2}, \ldots, z^{n}$ and $y^{1}, y^{2}, \ldots, y^{n}$ satisfying (17). Note that $z^{0} \in \mathbb{B}_{\alpha}(\bar{x})$ and $y^{0} \in \mathbb{B}_{\alpha}(\bar{y})$. We will first show that $z^{i} \in \mathbb{B}_{\alpha}(\bar{x})$ 
and $y^{i} \in \mathbb{B}_{\alpha}(\bar{y})$ for all $i=1,2, \ldots, n$. Indeed for $i=1$ this follows from (14) and (16). Utilizing (17), for $i \geq 2$ we have

$$
\begin{aligned}
& d_{X}\left(z^{i}, x\right) \leq \sum_{j=0}^{i-1} d_{X}\left(z^{j+1}, z^{j}\right) \\
& \leq \sum_{j=0}^{i-1}(\kappa(\mu+\varepsilon)+\varepsilon)^{j} d_{X}\left(z^{1}, x\right) \leq \frac{1}{1-(\kappa(\mu+\varepsilon)+\varepsilon)} d_{X}\left(z^{1}, x\right),
\end{aligned}
$$

and therefore, through (11) and (13),

$$
\begin{aligned}
d_{X}\left(z^{i}, \bar{x}\right) & \leq d_{X}\left(z^{i}, x\right)+d_{X}(x, \bar{x}) \\
& \leq \frac{a+\kappa b+\varepsilon}{1-(\kappa(\mu+\varepsilon)+\varepsilon)}+a \leq \alpha .
\end{aligned}
$$

Further, using (17) and (18),

$$
\begin{aligned}
d_{Y}\left(y^{i}, y^{0}\right) & \leq \sum_{j=0}^{i-1} d_{Y}\left(y^{j+1}, y^{j}\right) \\
& \leq \sum_{j=1}^{i-1}(\mu+\varepsilon) d_{X}\left(z^{j+1}, z^{j}\right) \leq \frac{\mu+\varepsilon}{1-(\kappa(\mu+\varepsilon)+\varepsilon)} d_{X}\left(z^{1}, x\right) .
\end{aligned}
$$

Hence, by (11),

$$
d_{Y}\left(y^{i}, \bar{y}\right) \leq \frac{(\mu+\varepsilon)(a+\kappa b+\varepsilon)}{1-(\kappa(\mu+\varepsilon)+\varepsilon)}+b \leq \alpha .
$$

If $z^{n}=z^{n-1}$, then $z^{n} \in F^{-1}\left(y^{n-1}\right)$ and $y^{n-1} \in \Psi\left(z^{n}\right)$. Hence $z^{n} \in F^{-1}\left(\Psi\left(z^{n}\right)\right)$. Then, through (12) and (18), we get

$$
\begin{aligned}
& d\left(x, \operatorname{Fix}\left(F^{-1} \Psi\right)\right) \leq d_{X}\left(x, z^{n}\right) \\
& \leq \frac{1}{1-(\kappa(\mu+\varepsilon)+\varepsilon)} d_{X}\left(z^{1}, x\right) \\
& \leq \frac{1}{1-(\kappa(\mu+\varepsilon)+\varepsilon)}[\kappa d(y, F(x))+\varepsilon] .
\end{aligned}
$$

Since the left side of this inequality does not depend on the $\varepsilon$ on the right and $y$ was arbitrarily chosen in $\Psi(x) \cap \mathbb{B}_{b}(\bar{y})$, by letting $\varepsilon$ go to 0 we obtain (8) with $U=\mathbb{B}_{a}(\bar{x})$ and $V=\mathbb{B}_{b}(\bar{y})$. Set $z^{n+1}=z^{n}, y^{n+1}=y^{n}$.

Assume next that $z^{n} \neq z^{n-1}$. Since $z^{n} \in F^{-1}\left(y^{n-1}\right) \cap \mathbb{B}_{\alpha}(\bar{x})$, from (9) there exists $z^{n+1} \in F^{-1}\left(y^{n}\right)$ such that

$$
\begin{aligned}
& d_{X}\left(z^{n+1}, z^{n}\right) \leq d\left(z^{n}, F^{-1}\left(y^{n}\right)\right)+\varepsilon d_{X}\left(z^{n}, z^{n-1}\right) \\
& \quad \leq \kappa d\left(y^{n}, F\left(z^{n}\right)\right)+\varepsilon d_{X}\left(z^{n}, z^{n-1}\right) \leq \kappa d_{Y}\left(y^{n}, y^{n-1}\right)+\varepsilon d_{X}\left(z^{n}, z^{n-1}\right) .
\end{aligned}
$$

Then, by invoking the induction hypothesis (17) for $k+1=n$,

$$
\begin{aligned}
& d_{X}\left(z^{n+1}, z^{n}\right) \leq \kappa(\mu+\varepsilon) d_{X}\left(z^{n}, z^{n-1}\right)+\varepsilon d_{X}\left(z^{n}, z^{n-1}\right) \\
& \quad=(\kappa(\mu+\varepsilon)+\varepsilon) d_{X}\left(z^{n}, z^{n-1}\right) \leq(\kappa(\mu+\varepsilon)+\varepsilon)^{n} d_{X}\left(z^{1}, x\right) .
\end{aligned}
$$

By repeating the argument in (19), if $z^{n+1}=z^{n}$, we get (8). Then set $y^{n+1}=y^{n}$. If $z^{n+1} \neq z^{n}$, since $y^{n} \in \Psi\left(z^{n}\right) \cap \mathbb{B}_{\alpha}(\bar{y})$, by (10), there exists $y^{n+1} \in \Psi\left(z^{n+1}\right)$ such that

$$
d_{Y}\left(y^{n+1}, y^{n}\right) \leq(\mu+\varepsilon) d_{X}\left(z^{n+1}, z^{n}\right) .
$$

This completes the induction step, and hence (17) holds for all $k$. 
We have already shown that if $z^{k}=z^{k-1}$ for some $k$, then (8) holds true. Suppose now that $z^{k+1} \neq z^{k}$ for all $k$. By virtue of the inequality for $z^{k+1}$ in (17), we see for any natural $n$ and $m$ with $m<n$ that

$$
\begin{aligned}
d_{X}\left(z^{n}, z^{m}\right) & \leq \sum_{k=m}^{n-1} d_{X}\left(z^{k+1}, z^{k}\right) \leq \sum_{k=m}^{n-1}(\kappa(\mu+\varepsilon)+\varepsilon)^{k} d_{X}\left(z^{1}, x\right) \\
& \leq \frac{d_{X}\left(z^{1}, x\right)}{1-(\kappa(\mu+\varepsilon)+\varepsilon)}(\kappa(\mu+\varepsilon)+\varepsilon)^{m} .
\end{aligned}
$$

But then, from (19) and the above inequalities,

$$
\begin{aligned}
d_{Y}\left(y^{n}, y^{m}\right) & \leq \sum_{k=m}^{n-1} d_{Y}\left(y^{k+1}, y^{k}\right) \leq \sum_{k=m}^{n-1}(\mu+\varepsilon) d_{X}\left(z^{k+1}, z^{k}\right) \\
& \leq \frac{(\mu+\varepsilon) d_{X}\left(z^{1}, x\right)}{1-(\kappa(\mu+\varepsilon)+\varepsilon)}(\kappa(\mu+\varepsilon)+\varepsilon)^{m} .
\end{aligned}
$$

This gives us that both sequences $\left\{z^{k}\right\}$ and $\left\{y^{k}\right\}$ satisfy the Cauchy condition and $z^{k} \in \mathbb{B}_{\alpha}(\bar{x}), y^{k} \in \mathbb{B}_{\alpha}(\bar{y})$ for all $k \geq 1$. Suppose that $\operatorname{gph} F \cap\left(\mathbb{B}_{\alpha}(\bar{x}) \times \mathbb{B}_{\alpha}(\bar{y})\right)$ is complete. Since $\left(z^{k}, y^{k-1}\right) \in \operatorname{gph} F \cap\left(\mathbb{B}_{a}(\bar{x}) \times \mathbb{B}_{\alpha}(\bar{y})\right)$ the sequence is convergent to, say, $(z, u) \in \operatorname{gph} F$. Note that $\left(z^{k}, y^{k}\right) \in \operatorname{gph} \Psi \times\left(\mathbb{B}_{\alpha}(\bar{x}) \times \mathbb{B}_{\alpha}(\bar{y})\right)$ which by assumption is closed and then $(z, u) \in \operatorname{gph} \Psi$. But then $z \in \operatorname{Fix}\left(F^{-1} \Psi\right)$. Now let gph $\Psi \cap\left(\mathbb{B}_{\alpha}(\bar{x}) \times \mathbb{B}_{\alpha}(\bar{y})\right)$ be complete. Since $\left(z^{k}, y^{k}\right) \in \operatorname{gph} \Psi \cap\left(\mathbb{B}_{\alpha}(\bar{x}) \times\right.$ $\left.\mathbb{B}_{\alpha}(\bar{y})\right)$ this sequence converges, and then, as in the preceding case, we conclude that $z \in \operatorname{Fix}\left(F^{-1} \Psi\right)$. Finally, if $\operatorname{gph} F^{-1} \Psi \cap\left(\mathbb{B}_{\alpha}(\bar{x}) \times \mathbb{B}_{\alpha}(\bar{x})\right)$ is complete, noting that $\left(z^{k}, z^{k+1}\right) \in \operatorname{gph} F^{-1} \Psi \cap\left(\mathbb{B}_{\alpha}(\bar{x}) \times \mathbb{B}_{\alpha}(\bar{x})\right)$, we obtain convergence of $\left\{z^{k}\right\}$ to a point $z \in \operatorname{Fix}\left(F^{-1} \Psi\right)$.

Utilizing (12) and (18), we finally obtain

$$
\begin{aligned}
d\left(x, \operatorname{Fix}\left(F^{-1} \Psi\right)\right) & \leq d_{X}(z, x)=\lim _{k \rightarrow \infty} d_{X}\left(z^{k}, x\right) \\
& \leq \frac{1}{1-(\kappa(\mu+\varepsilon)+\varepsilon)} d_{X}\left(z^{1}, x\right) \\
& \leq \frac{1}{1-(\kappa(\mu+\varepsilon)+\varepsilon)}[\kappa d(y, F(x))+\varepsilon] .
\end{aligned}
$$

Since $y$ was arbitrarily chosen in $\Psi(x) \cap \mathbb{B}_{b}(\bar{y})$, taking the limit as $\varepsilon \rightarrow 0$ gives us (8) with $U=\mathbb{B}_{a}(\bar{x})$ and $V=\mathbb{B}_{b}(\bar{y})$.

If the assumptions for $F$ and $\Psi$ hold globally, then we can take any $\bar{x} \in X$ and $\bar{y} \in Y$, and choosing $\alpha=+\infty$ repeat the proof with $a=b=+\infty$.

In the following theorem we present a slight extension of Theorem 5 in which the reference points for the mappings $F$ and $\Psi$ are different and which can be proved in a way parallel to the proof of Theorem 5 with only minor adjustments.

Theorem 6. Let $\left(X, d_{X}\right),\left(Y, d_{Y}\right)$ be metric spaces and $\alpha, \kappa$ and $\mu$ be positive constants such that $\kappa \mu<1$. Consider any two mappings $F: X \rightrightarrows Y$ and $\Psi$ : $X \rightrightarrows Y$ and points $(\bar{x}, \bar{y}) \in \operatorname{gph} F$ and $(\overline{\bar{x}}, \overline{\bar{y}}) \in \operatorname{gph} \Psi$ such that

$$
d_{Y}(\bar{y}, \overline{\bar{y}}) \leq \alpha / 2 \quad \text { and } \quad U:=\mathbb{B}_{\alpha}(\bar{x}) \cap \mathbb{B}_{\alpha}(\overline{\bar{x}}) \neq \emptyset .
$$

Assume that either one of the sets gph $F \cap\left(U \times \mathbb{B}_{\alpha}(\bar{y})\right)$ and $\operatorname{gph} \Psi \cap\left(U \times \mathbb{B}_{\alpha}(\overline{\bar{y}})\right)$ is complete while the other is closed or the set $\operatorname{gph}\left(F^{-1} \Psi\right) \cap(U \times U)$ is complete. Also assume that $F$ is metrically regular at $\bar{x}$ for $\bar{y}$ with constant $\kappa$ and neighborhoods 
$\mathbb{B}_{\alpha}(\bar{x})$ and $\mathbb{B}_{\alpha}(\bar{y})$, that is, (9) holds, and assume that $\Psi$ is Aubin continuous at $\overline{\bar{x}}$ for $\overline{\bar{y}}$ with constant $\mu$ and neighborhoods $\mathbb{B}_{\alpha}(\overline{\bar{x}})$ and $\mathbb{B}_{\alpha}(\overline{\bar{y}})$; that is,

$$
e\left(\Psi(x) \cap \mathbb{B}_{\alpha}(\overline{\bar{y}}), \Psi\left(x^{\prime}\right)\right) \leq \mu d_{X}\left(x, x^{\prime}\right) \quad \text { for all }\left(x, x^{\prime}\right) \in \mathbb{B}_{\alpha}(\overline{\bar{x}}) .
$$

Let $a$ and $b$ be any positive reals that satisfy

$$
\max \left\{\frac{a+\kappa b}{1-\kappa \mu}+a, 2\left[\frac{\mu a+\mu \kappa b}{1-\kappa \mu}+b\right]\right\}<\alpha .
$$

Then for any $x \in \mathbb{B}_{a}(\bar{x}) \cap \mathbb{B}_{a}(\overline{\bar{x}})$,

$$
d\left(x, \operatorname{Fix}\left(F^{-1} \Psi\right)\right) \leq \frac{\kappa}{1-\kappa \mu} d\left(\Psi(x) \cap \mathbb{B}_{b}(\bar{y}) \cap \mathbb{B}_{b}(\overline{\bar{y}}), F(x)\right) .
$$

Next comes a version of Theorem 5 which covers the case when $\Psi$ depends on a parameter, which can again be proved by slightly modifying the proof of Theorem 5 in order to take into account the dependence of the parameter.

Theorem 7. Let $\left(X, d_{X}\right),\left(Y, d_{Y}\right)$ be metric spaces, let $P$ be a metric space, and let $\kappa$ and $\mu$ be positive constants such that $\kappa \mu<1$. Consider any two mappings $F$ : $X \rightrightarrows Y$ and $\Psi: P \times X \rightrightarrows Y$, a point $\bar{p} \in P$ and a point $(\bar{x}, \bar{y}) \in \operatorname{gph} F \cap \operatorname{gph} \Psi(\bar{p}, \cdot)$ such that the following conditions hold:

(i) for any $p$ near $\bar{p}$, either one of the sets $\operatorname{gph} F$ and $\operatorname{gph} \Psi(p, \cdot)$ is locally complete while the other is locally closed at $(\bar{x}, \bar{y})$, or the set $\operatorname{gph} F^{-1}(\Psi(p, \cdot))$ is locally complete at $(\bar{x}, \bar{x})$;

(ii) $F$ is metrically regular at $\bar{x}$ for $\bar{y}$ with $\operatorname{reg}(F ; \bar{x} \mid \bar{y})<\kappa$;

(iii) $\Psi$ is partially Aubin continuous with respect to $x$ uniformly in $p$ at $(\bar{p}, \bar{x})$ for $\bar{y}$ with $\widehat{\operatorname{Lip}}_{x}(\Psi ;(\bar{p}, \bar{x}) \mid \bar{y})<\mu<1 / \kappa$.

Then there exist neighborhoods $Q$ of $\bar{p}, U$ of $\bar{x}$ and $V$ of $\bar{y}$ such that for any $p \in Q$ and $x \in U$,

$$
d\left(x, \operatorname{Fix}\left(F^{-1}(\Psi(p, \cdot))\right)\right) \leq \frac{\kappa}{1-\kappa \mu} d(\Psi(p, x) \cap V, F(x)) .
$$

Now we will derive Theorems 1-4 stated in Section 1.

Proof I of Theorem 1. For a function $g$ as in the statement, the local Lipschitz continuity of $g$ at $\bar{x}$ implies that, for some $r>0$, the graph of the restriction $g_{\mid \boldsymbol{B}_{r}(\bar{x})}$ is closed.

Pick $\alpha>0, \tilde{\kappa}>\kappa, \tilde{\mu}>\mu$ such that $\tilde{\kappa} \tilde{\mu}<1$,

$$
d\left(x, F^{-1}(y)\right) \leq \tilde{\kappa} d(y, F(x)) \text { for all }(x, y) \in \mathbb{B}_{\alpha}(\bar{x}) \times \mathbb{B}_{\alpha}(\bar{y})
$$

and

$$
d_{Y}\left(g(x), g\left(x^{\prime}\right)\right) \leq \tilde{\mu} d_{X}\left(x, x^{\prime}\right) \text { for all } x, x^{\prime} \in \mathbb{B}_{\alpha}(\bar{x}) .
$$

Without loss of generality, let $g(\bar{x})=0$. Choose positive $a$ and $b$ such that (21) holds with $\kappa, \mu$ replaced by $\tilde{\kappa}$ and $\tilde{\mu}$ respectively and in addition $a \tilde{\mu}<b$. Let $\gamma>0$ satisfy $a \tilde{\mu}+\gamma \leq b$ and $\gamma \leq \alpha / 2$. Pick $p \in \mathbb{B}_{\gamma}(\bar{y})$ and apply Theorem 6 for $F$ and $\Psi(x)=-g(x)+p$, with the so-chosen $\alpha, a$ and $b$, and with $\overline{\bar{y}}=p$ and $\overline{\bar{x}}=\bar{x}$. Then $x \in \operatorname{Fix}\left(F^{-1} \Psi\right)$ iff $x \in F^{-1}(-g(x)+p)$, which is the same as $x \in(F+g)^{-1}(p)$. Thus, from (22), for every $x \in \mathbb{B}_{a}(\bar{x})$,

$$
d\left(x,(F+g)^{-1}(p)\right) \leq \frac{\tilde{\kappa}}{1-\tilde{\kappa} \tilde{\mu}} d\left((-g(x)+p) \cap \mathbb{B}_{b}(\bar{y}) \cap \mathbb{B}_{b}(p), F(x)\right) .
$$

Since

$$
d_{Y}(-g(x)+p, \bar{y}) \leq d_{Y}(-g(x),-g(\bar{x}))+d_{Y}(p, \bar{y}) \leq \tilde{\mu} d_{X}(x, \bar{x})+\gamma \leq \tilde{\mu} a+\gamma<b
$$


and

$$
d_{Y}(-g(x)+p, p) \leq d_{Y}(-g(x),-g(\bar{x})) \leq \tilde{\mu} a \leq b,
$$

the intersection $(-g(x)+p) \cap \mathbb{B}_{b}(\bar{y}) \cap \mathbb{B}_{b}(p)$ is nonempty and hence consists of just the point $-g(x)+p$. But then the right side of $(24)$ equals $d(p,(g+F)(x))$. Since $\tilde{\kappa}$ and $\tilde{\mu}$ can be arbitrarily close to $\kappa$ and $\mu$, respectively, the proof is complete.

Proof II of Theorem 1. We will now derive Theorem 1 from the parametric Theorem 7. As in the preceding proof, let $g(\bar{x})=0$. We apply Theorem 7 with $\Psi(p, x)=-g(x)+p$ and $\bar{p}=\bar{y}$. Then $\widehat{\operatorname{Lip}}_{x}(\Psi ;(\bar{p}, \bar{x}) \mid \bar{y})=\operatorname{lip}(g ; \bar{x})$. Choosing $\tilde{\kappa}$ and $\tilde{\mu}$ as in Proof I, we obtain from Theorem 7 the existence of neighborhoods $Q$ of $\bar{p}, U$ of $\bar{x}$ and $V$ of $\bar{y}$ such that (23) holds for any $p \in Q$ and $x \in U$. Noting that $\operatorname{Fix}\left(F^{-1}(-g(\cdot)+p)\right)=(F+g)^{-1}(p)$ and, adjusting $V$ if necessary so that for any $x \in U, p \in Q$ the intersection $(-g(x)+p) \cap V$ is nonempty and hence consists of the single point $-g(x)+p$, we obtain from (23) that for any $x \in U, p \in Q$,

$$
d\left(x,(F+g)^{-1}(p)\right) \leq \frac{\tilde{\kappa}}{1-\tilde{\kappa} \tilde{\mu}} d(p,(g+F)(x)) .
$$

Since $\tilde{\kappa}$ and $\tilde{\mu}$ can be arbitrarily close to $\kappa$ and $\mu$, the proof follows.

Proof of Theorem 2. Apply Theorem 6 with $Y=X, \Psi=\Phi, \alpha=2 c, \mu=\lambda$, $\bar{y}=\overline{\bar{y}}=\bar{x}=\overline{\bar{x}}$ and $F$ the identity. Fix $\varepsilon>0$ such that $d(\bar{x}, \Phi(\bar{x}))+\varepsilon<\frac{\alpha}{2}(1-\mu)$ and define $b=d(\bar{x}, \Phi(\bar{x}))+\varepsilon$. Then $\Phi(\bar{x}) \cap \mathbb{B}_{b}(\bar{x}) \neq \emptyset$. Pick any $a>0$ satisfying

$$
a+b<\frac{\alpha}{2}(1-\mu)
$$

Then condition (21) holds and we can apply Theorem 6 , obtaining for $x=\bar{x}$ that

$$
d(\bar{x}, \operatorname{Fix}(\Phi)) \leq \frac{1}{1-\mu} \inf _{z \in \Phi(\bar{x}) \cap \boldsymbol{B}_{b}(\bar{x})} d_{X}(z, \bar{x}) .
$$

By the choice of $b$, the set $\Phi(\bar{x}) \cap \mathbb{B}_{b}(\bar{x})$, over which the infimum is taken on the right, is nonempty; hence

$$
d(\bar{x}, \operatorname{Fix}(\Phi)) \leq \frac{b}{1-\mu}<\frac{\alpha}{2}=c .
$$

Therefore $\Phi$ has a fixed point in $\mathbb{B}_{c}(\bar{x})$.

Proof of Theorem 3. Fix $z \in Y$. We apply Theorem 5 to $\Psi(\cdot)=-G(\cdot)+z$. If $x \in X$ is such that $F(x)+G(x)=\emptyset$, then $d(z,(F+G)(x))=\infty$ and therefore $d\left(x, \operatorname{Fix}\left(F^{-1} \Psi\right)\right) \leq \frac{\kappa}{1-\kappa \mu} d(z, G(x)+F(x))$. If both $F(x)$ and $G(x)$ are nonempty, applying Theorem 5 in the global case with $\Psi(x)=-G(x)+z$, we get

$$
\begin{aligned}
d\left(x, \operatorname{Fix}\left(F^{-1} \Psi\right)\right) & \leq \frac{\kappa}{1-\kappa \mu} \inf _{v \in G(x)} d(z-v, F(x)) \\
& =\frac{\kappa}{1-\kappa \mu} d(z, G(x)+F(x)) .
\end{aligned}
$$

Note that $x \in F^{-1}(-G(x)+z)$ is equivalent to the existence of $y \in F(x)$ and $v \in-G(x)$ such that $y=v+z$, which, in turn, is equivalent to $z=y-v \in$ $F(x)-v \in F(x)+G(x)$. Hence $\operatorname{Fix}\left(F^{-1}(-G(\cdot)-z)\right)=(F+G)^{-1}(z)$ and then, by $(25)$,

$$
d\left(x,(F+G)^{-1}(z)\right) \leq \frac{\kappa}{1-\kappa \mu} d(z,(F+G)(x)),
$$

which completes the proof. 
Proof of Theorem 4. From the global version of Theorem 5 with $\kappa=\gamma^{-1}$, for any $x \in X$ we have

$$
d\left(x, \operatorname{Fix}\left(F^{-1}(\Psi)\right)\right) \leq \frac{1}{\gamma-\mu} d(\Psi(x), F(x)) .
$$

It remains to note that for any $\varepsilon>0$ there is a point $\xi \in \operatorname{Fix}\left(F^{-1} \Psi\right)$ with $d_{X}(\xi, x) \leq$ $d\left(x, \operatorname{Fix}\left(F^{-1}(\Psi)\right)\right)+\varepsilon$, that is $F(\xi) \cap \Psi(\xi) \neq \emptyset$, and hence $\xi$ satisfies $(7)$.

One may argue that it is possible to obtain the local results displayed above from the corresponding global ones by using a truncation of the mapping in question. Specifically, for an Aubin continuous mapping, the question is whether one can find a submapping of it which would be Lipschitz continuous. First, observe that if a mapping $F$ is metrically regular, its restriction

$$
F_{\mid \boldsymbol{B}_{\alpha}(\bar{x})}(x)= \begin{cases}F(x) & \text { if } x \in \mathbb{B}_{\alpha}(\bar{x}) \\ \emptyset & \text { otherwise }\end{cases}
$$

may be not metrically regular on $\mathbb{B}_{\alpha}(\bar{x})$. Then, e.g., the global linear openness assumption of Theorem 4 may be lost by taking a restriction.

In [4] it was shown that when a mapping $\Psi$ acting in finite-dimensional spaces is convex-valued and is Aubin continuous at $\bar{x}$ for $\bar{y}$ with constant $\kappa$, then, for some positive $\alpha$ and $\beta$, the truncated mapping $\mathbb{B}_{\alpha}(\bar{x}) \ni x \mapsto \Psi(x) \cap \mathbb{B}_{\beta}(\bar{y})$ is Lipschitz continuous in $\mathbb{B}_{\alpha}(\bar{x})$ with Lipschitz constant larger than $\kappa$. In particular, taking truncation may increase the Lipschitz constant and lead to a violation of the inequality $\kappa \mu<1$.

The following example shows that in general Aubin continuous mappings may not be Lipschitz continuous after truncation. This example illustrates the essential difference between the global and the local cases.

Example 1. For all $i \geq 1$ define

$$
K_{i}=\left\{\left(z,\left(z-\frac{1}{2^{i}}\right)^{2}+\frac{1}{2^{i}}\right) \mid z \in\left[\frac{1}{2^{i}}, \frac{1}{2^{i-1}}\right]\right\}
$$

and let

$$
K=\{(0,0)\} \cup \bigcup_{i=1}^{\infty} K_{i} .
$$

Then $K$ is closed. Consider $\Psi: \mathbb{R} \rightrightarrows \mathbb{R}^{2}$ defined by

$$
\Psi(x)= \begin{cases}\left\{\left(x, \frac{1}{x}\right)\right\} \cup(x(0,1)+K) & \text { for } x>0, \\ K & \text { otherwise. }\end{cases}
$$

Then $\Psi$ is Aubin continuous at 0 for 0 with a constant 1 but $\Psi$ is not Lipschitz continuous in the sense of the Pompeiu-Hausdorff distance.

Moreover, observe that the mapping $x \rightrightarrows \Psi(x) \cap\left[-\frac{1}{2^{i}}, \frac{1}{2^{i}}\right] \times\left[-\frac{1}{2^{i}}, \frac{1}{2^{i}}\right]$ is not Lipschitz continuous, either. Furthermore, if $\left.a \in] 2^{-i-1}, 2^{-i}\right]$ and $\left.b \in\right] 2^{-i-1}, 4^{-i-1}+$ $\left.2^{-i-1}\right]$, then $x \rightrightarrows \Psi(x) \cap[-a, a] \times[-b, b]$ is Lipschitz continuous, but its Lipschitz constant increases to $\infty$ when $i \rightarrow+\infty$. 


\section{A PRoof of The EXtended LyUsterniK-GRAVES THEOREM THROUGH THE EKELAND PRINCIPLE}

In this section we provide a proof of Theorem 1, which is based on the Ekeland variational principle, for the case that $Y$ is a normed linear metric space. Applications of the Ekeland principle to proving Lyusternik-Graves type theorems under some additional conditions are discussed in [14].

Theorem 8 (Ekeland variational principle). Let $\left(X, d_{X}\right)$ be a complete metric space and let $f: X \rightarrow(-\infty, \infty]$ be a lower semicontinuous function on $X$ which is bounded from below. Let $\bar{u} \in \operatorname{dom} f$. Then for every $\delta>0$ there exists $u_{\delta}$ such that

$$
f\left(u_{\delta}\right)+\delta d_{X}\left(u_{\delta}, \bar{u}\right) \leq f(\bar{u})
$$

and

$$
f\left(u_{\delta}\right)<f(u)+\delta d_{X}\left(u, u_{\delta}\right) \text { for everyu } \in X, u \neq u_{\delta} .
$$

Proof of Theorem 1 for normed $Y$. It will be convenient to use the openness with linear rate (3) which is equivalent to metric regularity. Let $\kappa$ and $\mu$ be as in the statement of the theorem and then let $\tau$ and $\gamma$ be such that $1 / \tau>\kappa$ and $\mu<\gamma<\tau$. Then there exist positive $a, b$ and $h_{0}$ such that for all $(x, y) \in$ $\operatorname{gph} F \cap\left(\mathbb{B}_{a}(\bar{x}) \times \mathbb{B}_{b}(\bar{y})\right)$ and $h \in\left(0, h_{0}\right)$ we have

$$
y+h \tau \stackrel{\circ}{\mathbb{B}} \subset F\left(\stackrel{\circ}{B}_{h}(x)\right),
$$

and $g p h F \cap\left(\mathbb{B}_{a}(\bar{x}) \times \mathbb{B}_{b}(\bar{y})\right)$ is complete.

Let $g: X \rightarrow Y$ satisfy $\operatorname{lip}(g ; \bar{x}) \leq \mu<\gamma$ and then adjust $a$ if necessary so that

$$
\left\|g(x)-g\left(x^{\prime}\right)\right\| \leq \gamma d_{X}\left(x, x^{\prime}\right) \text { for all } x, x^{\prime} \in \mathbb{B}_{a}(\bar{x}) .
$$

Choose the positive constants $\alpha, \beta$ and $h_{1} \leq h_{0}$ so that

$$
\alpha+2 h_{1} \leq a \text { and } 2 \tau h_{1}+\beta \leq b .
$$

Fix $(x, y) \in \operatorname{gph} F \cap\left(\mathbb{B}_{\alpha}(\bar{x}) \times \mathbb{B}_{\beta}(\bar{y})\right)$ and any $h \in\left(0, h_{1}\right)$. We will now show that for any

$$
u \in y+g(x)+h(\tau-\gamma) \stackrel{\circ}{\mathbb{B}}
$$

one has $u \in(F+g)\left(\stackrel{\circ}{B}_{h}(x)\right)$. Fix $u$ as in (30). Then for some $\lambda>0$ one has

$$
u \in y+g(x)+\frac{h(\tau-\gamma)}{1+\lambda} \stackrel{\circ}{B} .
$$

It is sufficient to consider the case $u \neq y+g(x)$. Let $\theta>0$ be such that

$$
\|y+g(x)-u\|=\frac{(\tau-\gamma) h \theta^{2}}{1+\lambda} .
$$

Then clearly $0<\theta<1$. We apply the Ekeland principle (Theorem 8 above) on the complete metric space $K=\operatorname{gph} F \cap\left(\mathbb{B}_{h}(x) \times \mathbb{B}_{b}(\bar{y})\right)$ equipped with the metric

$$
d\left((x, y),\left(x^{\prime}, y^{\prime}\right)\right)=d_{X}\left(x, x^{\prime}\right)+\frac{\lambda}{\tau}\left\|y-y^{\prime}\right\|
$$

to the continuous function

$$
K \ni(\xi, \eta) \mapsto\|\eta+g(\xi)-u\|
$$


and to

$$
\bar{u}=(x, y) \quad \text { and } \quad \delta=\frac{(\tau-\gamma) \theta}{1+\lambda} .
$$

Let $u_{\delta}=(t, z)$ be as in the Ekeland principle. Then from (26)

$$
\|z+g(t)-u\|+\frac{(\tau-\gamma) \theta}{1+\lambda} d((t, z),(x, y)) \leq\|y+g(x)-u\|=\frac{(\tau-\gamma) h \theta^{2}}{1+\lambda}
$$

In particular, $d_{X}(t, x) \leq h \theta$. Hence, by $(29)$,

$$
d_{X}(t, \bar{x}) \leq \alpha+h \theta<a
$$

and also

$$
\begin{aligned}
\|z-\bar{y}\| & \leq\|z-y\|+\beta \leq\|z+g(t)-u\|+\|u-g(t)-y\|+\beta \\
& \leq\|z+g(t)-u\|+\|y+g(x)-u\|+\gamma d_{X}(t, x)+\beta \\
& \leq 2 \frac{\tau-\gamma}{1+\lambda} h+\gamma h+\beta<(2 \tau-\gamma) h+\beta<2 \tau h+\beta<b .
\end{aligned}
$$

Moreover, from (27), for any $\left(x^{\prime}, y^{\prime}\right) \in K$,

$$
\|z+g(t)-u\| \leq\left\|y^{\prime}+g\left(x^{\prime}\right)-u\right\|+\frac{(\tau-\gamma) \theta}{1+\lambda} d\left((t, z),\left(x^{\prime}, y^{\prime}\right)\right) .
$$

We will now show that $u=z+g(t)$. Indeed, assume $\|z+g(t)-u\|>0$, choose $\varepsilon \in(0, \tau)$ such that

$$
1-\frac{\varepsilon}{\tau-\gamma}>\theta
$$

and denote

$$
w=-\frac{\tau-\varepsilon}{\|z+g(t)-u\|}(z+g(t)-u) .
$$

Then, from (28), taking into account (31) and (32),

$$
z+h w \in F\left(B_{h}(t)\right) \text {. }
$$

This implies that

$$
z+h w \in F(\tilde{x}) \text { for some } \tilde{x} \in \mathbb{B}_{h}(t) .
$$

Note that, from (29) and (31),

$$
d_{X}(\tilde{x}, \bar{x}) \leq d_{X}(t, \bar{x})+h<a .
$$

Hence, applying (33) with $x^{\prime}=\tilde{x}$ and $y^{\prime}=z+h w$,

$$
\begin{aligned}
\|z+g(t)-u\| & \leq\|z+h w+g(\tilde{x})-u\|+\frac{(\tau-\gamma) \theta}{1+\lambda}\left(h+h \frac{\lambda}{\tau}\|w\|\right) \\
& <\|z+h w+g(t)-u\|+\|g(t)-g(\tilde{x})\|+h \theta(\tau-\gamma) \\
& \leq\|z+h w+g(t)-u\|+\gamma h+h \theta(\tau-\gamma) \\
& =\|z+g(t)-u\|\left(1-\frac{(\tau-\varepsilon) h}{\|z+g(t)-u\|}\right)+\gamma h+h \theta(\tau-\gamma) .
\end{aligned}
$$

This gives us

$$
(\tau-\varepsilon) h<\gamma h+\theta(\tau-\gamma) h
$$


hence $\tau-\gamma-\varepsilon<\theta(\tau-\gamma)$, which contradicts the choice of $\varepsilon$ in (34). Thus we have $u=z+g(t)$. Therefore $F+g$ is metrically regular at $\bar{x}$ for $\bar{y}+g(\bar{x})$ with constant $(\tau-\gamma)^{-1}$. Since $1 / \tau$ can be arbitrarily close to $\kappa$ and $\gamma$ can be arbitrarily close to $\mu$, we obtain the desired result.

\section{REFERENCES}

[1] A. V. Arutyunov, Covering mappings in metric spaces, and fixed points, Dokl. Akad. Nauk 416 (2007) 151-155 (Russian). MR2450913

[2] J-P. Aubin, Lipschitz behavior of solutions to convex minimization problems, Mathematics of Oper. Res. 9 (1984) 87-111. MR736641 (86f:90119)

[3] S. Banach, Théorie des Opérations Linéaires, Monografie Matematyczne, Warszawa, 1932. English translation by North Holland, Amsterdam, 1987. MR.0880204 (88a:01065)

[4] D. N. Bessis, Yu. S. Ledyaev and R. B. Vinter, Dualization of the Euler and Hamiltonian inclusions, Nonlinear Analysis 43 (2001) 861-882. MR.1813512 (2002c:49036)

[5] A. V. Dmitruk, A. A. Milyutin and N. P. Osmolovski1, Ljusternik's theorem and the theory of the extremum, Uspekhi Mat. Nauk 35, no. 6 (216) (1980) 11-46 (Russian). MR601755 (82c:58010)

[6] A. V. Dmitruk, On a nonlocal metric regularity of nonlinear operators, Control Cybernet. 34 (2005) 723-746. MR2208969 (2006k:49046)

[7] A. V. Dmitruk and A. Y. Kruger, Metric regularity and systems of generalized equations, J. Math. Anal. Appl. 342 (2008) 864-873. MR2445245 (2009f:49017)

[8] A. L. Dontchev and W. W. Hager, An inverse mapping theorem for set-valued maps, Proceedings of Amer. Math. Soc. 121 (1994) 481-489. MR1215027 (94h:58020)

[9] A. L. Dontchev and R. T. Rockafellar, Implicit Functions and Solution Mappings, Springer Mathematics Monographs, Springer, Dordrecht, 2009. MR2515104

[10] A. L. Dontchev, A. S. Lewis and R. T. Rockafellar, The radius of metric regularity, Trans. Amer. Math. Soc. 355 (2003) 493-517. MR.1932710 (2003i:49026)

[11] H. Frankowska, Some inverse mapping theorems, Annales de Inst. H. Poincaré Anal. Non Linéaire, Section C, 7 (1990) 183-234. MR1065873 (91j:49020)

[12] H. Frankowska, Conical inverse mapping theorems, Bull. Austral. Math. Soc. 45 (1992) 5360. MR 1147244 (92k:49028)

[13] L. M. Graves, Some mapping theorems, Duke Math. Journal 17 (1950) 111-114. MR0035398 $(11: 729 \mathrm{e})$

[14] A. D. Ioffe, Metric regularity and subdifferential calculus, Uspekhi Mat. Nauk 55 (2000), no. 3 (333), 103-162; English translation in Math. Surveys 55 (2000), 501-558. MR1777352 $(2001 \mathrm{j}: 90002)$

[15] A. D. Ioffe, Towards variational analysis in metric spaces: metric regularity and fixed points, Math. Program., Ser. B 123 (2010) 241-252. MR2577330

[16] L. A. Lyusternik, On the conditional extrema of functionals, Mat. Sbornik 41 (1934) 390401.

[17] S. B. Nadler, Jr., Multi-valued contraction mapping, Pacific J. Math. 30 (1969) 475-488. MR0254828(40:8035)

Mathematical Reviews and the University of Michigan, Ann Arbor, Michigan 48109. On leave from the Institute of Mathematics, Bulgarian Academy of Sciences, Sofia, BULGARIA

E-mail address: ald@ams.org

Combinatoire \& Optimisation, CNRS, Université Pierre et Marie Curie, 4 place Jussieu, 75252 Paris, France

E-mail address: frankowska@math.jussieu.fr 